\title{
Bifurcation of limit cycles at degenerate singular point and infinity in a septic system
}

Yusen $\mathrm{Wu}^{1 *}$ and Feng $\mathrm{Li}^{2}$

\author{
*Correspondence: \\ wuyusen82714@yahoo.com.cn \\ ${ }^{1}$ School of Mathematics and \\ Statistics, Henan University of \\ Science and Technology, Luoyang, \\ Henan 471003, P.R. China \\ Full list of author information is \\ available at the end of the article
}

\begin{abstract}
In this paper, the problem of bifurcation of limit cycles from degenerate singular point and infinity in a class of septic polynomial differential systems is investigated. Using the computer algebra system Mathematica, the limit cycle configurations of $\{(8), 3\}$ and $\{(3), 6\}$ are obtained under synchronous perturbation at degenerate singular point and infinity. To our knowledge, up to now, this is the first time that the problem of limit cycles bifurcated from degenerate singular point and infinity under synchronous perturbed conditions in a septic system has been investigated.
\end{abstract}

MSC: $34 \mathrm{C} 05 ; 34 \mathrm{CO}$

Keywords: degenerate singular point; infinity; bifurcation of limit cycles; septic system

\section{Introduction}

In the qualitative theory of planar dynamical systems, bifurcation of limit cycles for planar polynomial differential system

$$
\frac{d x}{d t}=P(x, y), \quad \frac{d y}{d t}=Q(x, y),
$$

which belongs to the second part of the Hilbert 16th problem, is known as a hot but intractable issue. In the case of limit cycles bifurcated from the origin, a lot of work has been done (see $[1,2])$. Let $I_{n}$ be the maximum possible number of limit cycles in the neighborhood of the infinity (large amplitude limit cycles) of system (1.1) when $P(x, y), Q(x, y)$ are of degree at most $n$. As far as the number of limit cycles bifurcated from infinity is concerned, representative results are as follows: cubic systems, [3] got $I_{3} \geq 4$, [4] got $I_{3} \geq 5$, [5] got $I_{3} \geq 6$, [6,7] got $I_{3} \geq 7$; quintic systems, [8] got $I_{5} \geq 4$, [9] got $I_{5} \geq 6$, [10] got $I_{5} \geq 8$, [11] got $I_{5} \geq 11$; septic systems, [12] got $I_{7} \geq 8$, [13] got $I_{7} \geq 9$, [14] got $I_{7} \geq 13$.

Definition 1.1 [4] We call the configuration $\left\{\left(k_{1}\right), k_{2}\right\}$ the configuration of a vector field with $k_{1}$ 'small' limit cycles and $k_{2}$ 'large' ones.

Few papers are concerned with bifurcation of limit cycles from the origin and infinity under synchronous perturbation: [4] obtained the limit cycle configurations of $\{(5), 2\}$ and $\{(2), 4\}$ in a cubic polynomial differential system; [15] and [16] respectively got the limit cycle configurations of $\{(5), 2\},\{(2), 4\}$ and $\{(7), 2\},\{(2), 6\}$ in two certain quintic systems. However, for a higher degree system, there is not any result.

(c) 2013 Wu and Li; licensee Springer. This is an Open Access article distributed under the terms of the Creative Commons Attribution License (http://creativecommons.org/licenses/by/2.0), which permits unrestricted use, distribution, and reproduction in any medium, provided the original work is properly cited. 
In this paper, we deal with limit cycles bifurcated from degenerate singular point and infinity under synchronous perturbation in the following real septic polynomial differential system:

$$
\begin{aligned}
& \frac{d x}{d t}=\lambda\left(\delta_{1} x-y\right)\left(x^{2}+y^{2}\right)+X_{5}(x, y)+\left(\delta_{2} x-y\right)\left(x^{2}+y^{2}\right)^{3} \\
& \frac{d y}{d t}=\lambda\left(x+\delta_{1} y\right)\left(x^{2}+y^{2}\right)+Y_{5}(x, y)+\left(x+\delta_{2} y\right)\left(x^{2}+y^{2}\right)^{3}
\end{aligned}
$$

where $\lambda \neq 0$ and

$$
\begin{aligned}
& X_{5}(x, y)=\sum_{k+j=5} A_{k j} x^{k} y^{j}, \quad Y_{5}(x, y)=\sum_{k+j=5} B_{k j} x^{k} y^{j}, \\
& A_{50}=\beta_{03}+\beta_{12}+\beta_{21}+\beta_{30}, \quad B_{50}=\alpha_{03}+\alpha_{12}+\alpha_{21}+\alpha_{30}, \\
& A_{41}=-5 \alpha_{03}-3 \alpha_{12}-\alpha_{21}+\alpha_{30}, \quad B_{41}=5 \beta_{03}+3 \beta_{12}+\beta_{21}-\beta_{30}, \\
& A_{32}=-2\left(5 \beta_{03}+\beta_{12}-\beta_{21}-\beta_{30}\right), \quad B_{32}=-2\left(5 \alpha_{03}+\alpha_{12}-\alpha_{21}-\alpha_{30}\right), \\
& A_{23}=2\left(5 \alpha_{03}-\alpha_{12}-\alpha_{21}+\alpha_{30}\right), \quad B_{23}=-2\left(5 \beta_{03}-\beta_{12}-\beta_{21}+\beta_{30}\right), \\
& A_{14}=5 \beta_{03}-3 \beta_{12}+\beta_{21}+\beta_{30}, \quad B_{14}=5 \alpha_{03}-3 \alpha_{12}+\alpha_{21}+\alpha_{30}, \\
& A_{05}=-\alpha_{03}+\alpha_{12}-\alpha_{21}+\alpha_{30}, \quad B_{05}=\beta_{03}-\beta_{12}+\beta_{21}-\beta_{30} .
\end{aligned}
$$

This paper is organized as follows. In Section 2, some preliminaries are given and system (1.2) is transferred to its complex concomitant system. In Sections 3 and 4, the first nine singular point quantities at degenerate singular point and the first seven singular point quantities at infinity are deduced. At the same time, their center conditions are obtained. In Section 5, the sufficient conditions of limit cycle configurations $\{(8), 3\}$ and $\{(3), 6\}$ are presented.

\section{Conversion of the system}

Obviously, the origin of system (1.2) is either a center or a focus, and it is also a degenerate singular point. System (1.2) contains no real singular point on the equator $\Gamma_{\infty}$ of the Poincaré compactification on the sphere. $\Gamma_{\infty}$ is called infinity on the Gauss sphere or the equator of system (1.2).

By means of polar coordinate transformation

$$
x=r \cos \theta, \quad y=r \sin \theta,
$$

system (1.2) takes the form

$$
\frac{d r}{d \theta}=r \frac{\delta_{1} \lambda+\delta_{2} r^{4}+r^{2}\left[\cos \theta X_{5}(\cos \theta, \sin \theta)+\sin \theta Y_{5}(\cos \theta, \sin \theta)\right]}{\lambda+r^{4}+r^{2}\left[\cos \theta Y_{5}(\cos \theta, \sin \theta)-\sin \theta X_{5}(\cos \theta, \sin \theta)\right]} .
$$

For a sufficiently small $h$, let

$$
r=r(\theta, h)=\sum_{m=1}^{\infty} v_{m}(\theta) h^{m}
$$


be the solution of Eq. (2.2) satisfying the initial value condition $\left.r\right|_{\theta=0}=h$, where

$$
v_{1}(\theta)=e^{\delta_{1} \theta}, \quad v_{m}(0)=0, \quad m=2,3, \ldots
$$

The 0 th focal value at the origin of system $(1.2)$ is $v_{1}(2 \pi)-1$, i.e., $e^{2 \pi \delta_{1}}-1$, the $k$ th one is $\nu_{2 k+1}(2 \pi), k=1,2, \ldots$.

By means of generalized polar coordinate transformation

$$
x=r^{-1} \cos \theta, \quad y=r^{-1} \sin \theta,
$$

system (1.2) takes the form

$$
\frac{d r}{d \theta}=-r \frac{\delta_{2}+\delta_{1} \lambda r^{4}+r^{2}\left[\cos \theta X_{5}(\cos \theta, \sin \theta)+\sin \theta Y_{5}(\cos \theta, \sin \theta)\right]}{1+\lambda r^{4}+r^{2}\left[\cos \theta Y_{5}(\cos \theta, \sin \theta)-\sin \theta X_{5}(\cos \theta, \sin \theta)\right]} .
$$

For a sufficiently small $h$, let

$$
r=r(\theta, h)=\sum_{m=1}^{\infty} v_{m}(\theta) h^{m}
$$

be the solution of Eq. (2.6) satisfying the initial value condition $\left.r\right|_{\theta=0}=h$, where

$$
v_{1}(\theta)=e^{-\delta_{2} \theta}, \quad v_{m}(0)=0, \quad m=2,3, \ldots
$$

The 0 th focal value at infinity of system $(1.2)$ is $\nu_{1}(2 \pi)-1$, i.e., $e^{-2 \pi \delta_{2}}-1$, the $k$ th one is $\nu_{2 k+1}(2 \pi), k=1,2, \ldots$

By means of transformation

$$
z=x+i y, \quad w=x-i y, \quad T=i t, \quad i=\sqrt{-1},
$$

system (1.2) can be transformed into the following complex system:

$$
\begin{aligned}
& \frac{d z}{d T}=\lambda\left(1-i \delta_{1}\right) z^{2} w+b_{03} z^{5}+b_{12} z^{4} w+b_{21} z^{3} w^{2}+b_{30} z^{2} w^{3}+\left(1-i \delta_{2}\right) z^{4} w^{3} \\
& \frac{d w}{d T}=-\left[\lambda\left(1+i \delta_{1}\right) w^{2} z+a_{03} w^{5}+a_{12} w^{4} z+a_{21} w^{3} z^{2}+a_{30} w^{2} z^{3}+\left(1+i \delta_{2}\right) w^{4} z^{3}\right]
\end{aligned}
$$

where

$$
\begin{array}{ll}
a_{30}=\alpha_{30}+i \beta_{30}, & a_{21}=\alpha_{21}+i \beta_{21}, \\
a_{12}=\alpha_{12}+i \beta_{12}, & a_{03}=\alpha_{03}+i \beta_{03}, \\
b_{30}=\alpha_{30}-i \beta_{30}, & b_{21}=\alpha_{21}-i \beta_{21}, \\
b_{12}=\alpha_{12}-i \beta_{12}, & b_{03}=\alpha_{03}-i \beta_{03} .
\end{array}
$$

It is obvious that the coefficients of system (2.10) satisfy the conjugate condition, i.e.,

$$
b_{i j}=\overline{a_{i j}}, \quad(i, j) \in\{(3,0),(2,1),(1,2),(0,3)\} .
$$

We call that systems (1.2) and (2.10) are associated. 


\section{Singular point quantities and center conditions at degenerate singular point} From Theorem 3.2 in [3], we have

Lemma 3.1 For system $(2.10)_{\delta_{1}=\delta_{2}=0}$, we can determine uniquely an extended formal power series

$$
F(z, w)=z w \sum_{k=0}^{\infty} \frac{f_{5 k}(z, w)}{(z w)^{2 k}},
$$

such that

$$
\left.\frac{d F}{d T}\right|_{(2.10)_{\delta_{1}=\delta_{2}=0}}=\sum_{m=1}^{\infty} \mu_{m}^{(0)}(z w)^{m+2},
$$

where $c_{00}=1, c_{k k}=0, k=1,2, \ldots, f_{5 k}(z, w)=\sum_{\alpha+\beta=5 k} c_{\alpha \beta} z^{\alpha} w^{\beta}$, and for any positive integer $m$, the $m$ th singular point quantity at the origin $\mu_{m}^{(0)}$ can be determined by the following recursion formulas:

$$
\begin{aligned}
& c_{00}=1 \\
& \text { when }(\alpha=\beta>0) \text { or } \alpha<0 \text { or } \beta<0, c_{\alpha \beta}=0 ; \\
& \text { else } \\
& \begin{aligned}
c_{\alpha \beta}= & \frac{1}{5 \lambda(\beta-\alpha)}\left((5 \alpha-5 \beta) c_{-10+\alpha,-10+\beta}+b_{03}(-10+3 \alpha-2 \beta) c_{-7+\alpha,-3+\beta}\right. \\
& +\left(b_{12}(-5+3 \alpha-2 \beta)-a_{30}(5-2 \alpha+3 \beta)\right) c_{-6+\alpha,-4+\beta}+\left(b_{21}(3 \alpha-2 \beta)\right. \\
& \left.-a_{21}(-2 \alpha+3 \beta)\right) c_{-5+\alpha,-5+\beta}+\left(b_{30}(5+3 \alpha-2 \beta)-a_{12}(-5-2 \alpha+3 \beta)\right) \\
& \left.\times c_{-4+\alpha,-6+\beta}-a_{03}(-10-2 \alpha+3 \beta) c_{-3+\alpha,-7+\beta}\right), \\
\mu_{m}^{(0)}= & b_{03}(-2+m) c_{-17+5(2+m),-13+5(2+m)}+\left(b_{12}(-1+m)-a_{30}(1+m)\right) \\
& \times c_{-16+5(2+m),-14+5(2+m)}+\left(-a_{21} m+b_{21} m\right) c_{-15+5(2+m),-15+5(2+m)} \\
& +\left(-a_{12}(-1+m)+b_{30}(1+m)\right) c_{-14+5(2+m),-16+5(2+m)}-a_{03}(-2+m) \\
& \times c_{-13+5(2+m),-17+5(2+m) .} .
\end{aligned}
\end{aligned}
$$

Using the recursion formulas to do symbolic computation, we have

Theorem 3.1 The first nine singular point quantities at the origin of system $(2.10)_{\delta_{1}=\delta_{2}=0}$ are as follows:

$$
\begin{aligned}
& \mu_{1}^{(0)}=-a_{21}+b_{21} \\
& \mu_{2}^{(0)}=\frac{a_{12} a_{30}-b_{12} b_{30}}{\lambda}, \\
& \mu_{3}^{(0)}=-\frac{9 a_{03} a_{30}^{2}+a_{12}^{2} b_{03}-a_{03} b_{12}^{2}-9 b_{03} b_{30}^{2}}{8 \lambda^{2}}, \\
& \mu_{4}^{(0)}=-\frac{\left(a_{21}+b_{21}\right)\left(a_{12}^{2} b_{03}+3 a_{03} a_{30} b_{12}-a_{03} b_{12}^{2}-3 a_{12} b_{03} b_{30}\right)}{12 \lambda^{3}}, \\
& \mu_{5}^{(0)}=-\frac{\left(a_{12}^{2} b_{03}+3 a_{03} a_{30} b_{12}-a_{03} b_{12}^{2}-3 a_{12} b_{03} b_{30}\right)\left(-27 a_{03} b_{03}+16 a_{12} b_{12}+216 \lambda\right)}{648 \lambda^{4}},
\end{aligned}
$$




$$
\begin{aligned}
& \mu_{6}^{(0)}=0, \\
& \mu_{7}^{(0)}=-\frac{\left(405 a_{03}^{2} b_{03}^{2}-8,928 a_{03} a_{12} b_{03} b_{12}+6,400 a_{12}^{2} b_{12}^{2}\right)\left(-a_{12}^{2} b_{03}-3 a_{03} a_{30} b_{12}+a_{03} b_{12}^{2}+3 a_{12} b_{03} b_{30}\right)}{466,560 \lambda^{6}}, \\
& \mu_{8}^{(0)}=\frac{7\left(45 a_{03} b_{03}-32 a_{12} b_{12}\right)\left(a_{12}^{2} b_{03}+a_{03} b_{12}^{2}\right)\left(-a_{12}^{2} b_{03}-3 a_{03} a_{30} b_{12}+a_{03} b_{12}^{2}+3 a_{12} b_{03} b_{30}\right)}{69,984 \lambda^{7}}, \\
& \mu_{9}^{(0)}=\frac{11 a_{12}^{2} b_{12}^{2}\left(-224,181 a_{03} b_{03}+164,000 a_{12} b_{12}\right)\left(a_{12}^{2} b_{03}+3 a_{03} a_{30} b_{12}-a_{03} b_{12}^{2}-3 a_{12} b_{03} b_{30}\right)}{13,778,100 \lambda^{8}} .
\end{aligned}
$$

In the above expression of $\mu_{k}^{(0)}$, we have already let $\mu_{1}^{(0)}=\cdots=\mu_{k-1}^{(0)}=0, k=2,3, \ldots, 9$.

From Theorem 3.1, it is easy to get

Theorem 3.2 For system $(2.10)_{\delta_{1}=\delta_{2}=0}$, the first nine singular point quantities at the origin are zero if and only if one of the following conditions holds:

$$
\begin{array}{ll}
\text { (I) } & a_{21}=b_{21}, \quad b_{30}=\frac{1}{3} a_{12}, \quad a_{30}=\frac{1}{3} b_{12}, \quad a_{12} b_{12} \neq 0 ; \\
\text { (II) } & a_{21}=b_{21}, \quad a_{12} a_{30}=b_{12} b_{30}, \quad a_{12}^{2} b_{03}=b_{12}^{2} a_{03}, \\
& a_{12} b_{30} b_{03}=b_{12} a_{30} a_{03}, \quad b_{30}^{2} b_{03}=a_{30}^{2} a_{03} .
\end{array}
$$

Proof Putting condition (I) or (II) into expression (3.5) can easily complete the proof of sufficiency.

Now, let us prove the necessity. If $a_{12}=b_{12}=0$, then $\mu_{2}^{(0)}=\mu_{4}^{(0)}=\mu_{5}^{(0)}=\mu_{6}^{(0)}=\mu_{7}^{(0)}=$ $\mu_{8}^{(0)}=\mu_{9}^{(0)}=0, \mu_{3}^{(0)}=-\frac{9\left(a_{03} a_{30}^{2}-b_{03} b_{30}^{2}\right)}{8 \lambda^{2}}$. Therefore, $\mu_{k}^{(0)}=0(k=1,2, \ldots, 9)$ yields

$$
a_{21}=b_{21}, \quad a_{30}^{2} a_{03}=b_{30}^{2} b_{03}, \quad a_{12}=b_{12}=0 .
$$

If $a_{12} b_{12} \neq 0$, notice that $\mu_{1}^{(0)}=-a_{21}+b_{21}=0, \mu_{2}^{(0)}=\frac{a_{12} a_{30}-b_{12} b_{30}}{\lambda}=0$, there exist $r_{32}$ and $p$ such that

$$
a_{21}=b_{21}=r_{21}, \quad b_{30}=p a_{12}, \quad a_{30}=p b_{12} .
$$

Substituting (3.7) into expression (3.5), we get

$$
\begin{aligned}
& \mu_{3}^{(0)}=\frac{\left(a_{12}^{2} b_{03}-a_{03} b_{12}^{2}\right)(-1+3 p)(1+3 p)}{8 \lambda^{2}}, \\
& \mu_{4}^{(0)}=\frac{\left(a_{12}^{2} b_{03}-a_{03} b_{12}^{2}\right)(-1+3 p) r_{21}}{6 \lambda^{3}}, \\
& \mu_{5}^{(0)}=\frac{\left(a_{12}^{2} b_{03}-a_{03} b_{12}^{2}\right)(-1+3 p)\left(-27 a_{03} b_{03}+16 a_{12} b_{12}+216 \lambda\right)}{648 \lambda^{4}}, \\
& \mu_{7}^{(0)}=-\frac{\left(a_{12}^{2} b_{03}-a_{03} b_{12}^{2}\right)\left(405 a_{03}^{2} b_{03}^{2}-8,928 a_{03} a_{12} b_{03} b_{12}+6,400 a_{12}^{2} b_{12}^{2}\right)(-1+3 p)}{466,560 \lambda^{6}}, \\
& \mu_{8}^{(0)}=-\frac{7\left(-45 a_{03} b_{03}+32 a_{12} b_{12}\right)\left(a_{12}^{2} b_{03}-a_{03} b_{12}^{2}\right)\left(a_{12}^{2} b_{03}+a_{03} b_{12}^{2}\right)(-1+3 p)}{69,984 \lambda^{7}}, \\
& \mu_{9}^{(0)}=-\frac{11 a_{12}^{2} b_{12}^{2}\left(-224,181 a_{03} b_{03}+164,000 a_{12} b_{12}\right)\left(a_{12}^{2} b_{03}-a_{03} b_{12}^{2}\right)(-1+3 p)}{13,778,100 \lambda^{8}} .
\end{aligned}
$$

$405 a_{03}^{2} b_{03}^{2}-8,928 a_{03} a_{12} b_{03} b_{12}+6,400 a_{12}^{2} b_{12}^{2}=0$ and $-224,181 a_{03} b_{03}+164,000 a_{12} b_{12}=0$ do not simultaneously hold, thus from $\mu_{2}^{(0)}=\mu_{7}^{(0)}=\mu_{9}^{(0)}=0$, we have (i) $p=\frac{1}{3}$ or (ii) $a_{12} a_{30}-$ 
$b_{12} b_{30}=0, a_{12}^{2} b_{03}-a_{03} b_{12}^{2}=0$. At this moment, $\mu_{3}^{(0)}=\mu_{4}^{(0)}=\mu_{5}^{(0)}=\mu_{8}^{(0)}=0$. Condition (i) or (ii) combining with $\mu_{1}^{(0)}=0$ implies the following conditions, respectively:

$$
\begin{aligned}
& a_{21}=b_{21}, \quad b_{30}=\frac{1}{3} a_{12}, \quad a_{30}=\frac{1}{3} b_{12}, \quad a_{12} b_{12} \neq 0 ; \\
& a_{21}=b_{21}, \quad a_{12} a_{30}=b_{12} b_{30}, \quad a_{12}^{2} b_{03}=b_{12}^{2} a_{03}, \quad a_{12} b_{12} \neq 0 .
\end{aligned}
$$

Condition (3.6) plus (3.10) is equivalent to condition (II). The proof is completed.

From the definition of elementary Lie invariants in [17], we can obtain

Lemma 3.2 All the elementary Lie invariants of system $(2.10)_{\delta_{1}=\delta_{2}=0}$ are as follows:

$$
\begin{aligned}
& \lambda, \quad a_{21}, \quad b_{21}, \quad a_{30} b_{30}, \quad a_{12} b_{12}, \\
& a_{03} b_{03}, \quad a_{12} a_{30}, \quad b_{12} b_{30}, \\
& a_{12}^{2} b_{03}, \quad a_{12} b_{30} b_{03}, \quad b_{30}^{2} b_{03}, \\
& b_{12}^{2} a_{03}, \quad b_{12} a_{30} a_{03}, \quad a_{30}^{2} a_{03} \text {. }
\end{aligned}
$$

Theorem 3.3 For system $(2.10)_{\delta_{1}=\delta_{2}=0}$, all the singular point quantities at the origin are zero if and only if the first nine singular point quantities are zero, i.e., one of the two conditions in Theorem 3.2 holds. Correspondingly, the two conditions in Theorem 3.2 are the center conditions of the origin.

Proof When condition (I) holds, system $(2.10)_{\delta_{1}=\delta_{2}=0}$ has the integrating factor $M(z, w)=$ $(z w)^{-5}$; when condition (II) holds, system $(2.10)_{\delta_{1}=\delta_{2}=0}$ satisfies the conditions of the extended symmetric principle (Theorem 2.6 in [17]).

\section{Singular point quantities and center conditions at infinity}

Lemma 4.1 [18] For the system

$$
\begin{aligned}
& \frac{d z}{d T}=z^{n+1} w^{n}+\sum_{k=0}^{2 n} Z_{k}(z, w), \\
& \frac{d w}{d T}=-w^{n+1} z^{n}-\sum_{k=0}^{2 n} W_{k}(z, w),
\end{aligned}
$$

where

$$
Z_{k}(z, w)=\sum_{\alpha+\beta=k} a_{\alpha \beta} z^{\alpha} w^{\beta}, \quad W_{k}(z, w)=\sum_{\alpha+\beta=k} b_{\alpha \beta} w^{\alpha} z^{\beta},
$$

to any integer $s \neq 0, \gamma \neq 0$, we can derive successively the terms of the following formal series:

$$
F(z, w)=(z w)^{s}\left[\sum_{k=0}^{\infty} \frac{f_{(2 n+1) k}(z, w)}{(z w)^{k(n+1)}}\right]^{\frac{1}{\gamma}}
$$


such that

$$
\left.\frac{d F}{d T}\right|_{(4.1)}=\frac{1}{\gamma}(z w)^{n+s}\left[\sum_{k=0}^{\infty} \frac{f_{(2 n+1) k}(z, w)}{(z w)^{k(n+1)}}\right]^{\frac{1}{\gamma}-1} \sum_{m=1}^{\infty} \frac{\lambda_{m}}{(z w)^{m}},
$$

and for any integer $m$,

$$
\lambda_{m} \sim-s \gamma \mu_{m}
$$

where $\mu_{m}$ is the mth singular point quantity at infinity, ' ' is the symbol of algebraic equivalence, $c_{00}=1, c_{(2 n+1) k,(2 n+1) k}$ can be taken arbitrarily, $k=1,2, \ldots$ When $\alpha \neq \beta, \lambda_{m}$ is determined by the following recursion formulas:

$$
\begin{aligned}
c_{\alpha \beta}= & \frac{1}{(\beta-\alpha)(2 n+1)} \sum_{k, j}\left\{[n \alpha-(n+1) \beta+(\gamma s+n+1-k)(2 n+1)] a_{k, j-1}\right. \\
& \left.-[n \beta-(n+1) \alpha+(\gamma s+n+1-j)] b_{j, k-1}\right\} \\
& \times c_{\alpha+n k+(n+1) j-(n+1)(2 n+1), \beta+n j+(n+1) k-(n+1)(2 n+1),} \\
\lambda_{m}= & \sum_{k, j}\left[(\gamma s+n+1-k-m) a_{k, j-1}-(\gamma s+n+1-j-m) b_{j, k-1}\right] \\
& \times c_{n k+(n+1) j+(m-n-1)(2 n+1), n j+(n+1) k+(m-n-1)(2 n+1) .}
\end{aligned}
$$

Take $s=-1, \gamma=1, n=3$, applying Lemma 4.1 to system $(2.10)_{\delta_{1}=\delta_{2}=0}$ and executing calculation in Mathematica, we have

Theorem 4.1 The first seven singular point quantities at infinity of system $(2.10)_{\delta_{1}=\delta_{2}=0}$ are as follows:

$$
\begin{aligned}
& \mu_{1}^{(\infty)}=a_{21}-b_{21}, \\
& \mu_{2}^{(\infty)}=-a_{12} a_{30}+b_{12} b_{30}, \\
& \mu_{3}^{(\infty)}=\frac{3 a_{03} a_{30}^{2}+3 a_{12}^{2} b_{03}+8 a_{03} a_{30} b_{12}-3 a_{03} b_{12}^{2}-8 a_{12} b_{03} b_{30}-3 b_{03} b_{30}^{2}}{8}, \\
& \mu_{4}^{(\infty)}=-\frac{\left(a_{21}+b_{21}\right)\left(a_{12}^{2} b_{03}+3 a_{03} a_{30} b_{12}-a_{03} b_{12}^{2}-3 a_{12} b_{03} b_{30}\right)}{4}, \\
& \mu_{5}^{(\infty)}=-\frac{\left(a_{12}^{2} b_{03}+3 a_{03} a_{30} b_{12}-a_{03} b_{12}^{2}-3 a_{12} b_{03} b_{30}\right)\left(-a_{03} b_{03}+4 a_{12} b_{12}+6 \lambda\right)}{6}, \\
& \mu_{6}^{(\infty)}=0, \\
& \mu_{7}^{(\infty)}=\frac{5 a_{12} b_{12}\left(-a_{03} b_{03}+4 a_{12} b_{12}\right)\left(a_{12}^{2} b_{03}+3 a_{03} a_{30} b_{12}-a_{03} b_{12}^{2}-3 a_{12} b_{03} b_{30}\right)}{6} .
\end{aligned}
$$

In the above expression of $\mu_{k}^{(\infty)}$, we have already let $\mu_{1}^{(\infty)}=\cdots=\mu_{k-1}^{(\infty)}=0, k=2,3, \ldots, 7$.

By the similar method used in the proof of Theorem 3.1, we can obtain the following result.

Theorem 4.2 For system $(2.10)_{\delta_{1}=\delta_{2}=0}$, the first seven singular point quantities at infinity are zero if and only if condition (I) or (II) is satisfied. 
The following result is immediate from Theorems 3.3 and 4.2.

Theorem 4.3 The degenerate singular point and infinity of system $(2.10)_{\delta_{1}=\delta_{2}=0}$ have the same center conditions.

\section{Bifurcation of limit cycles under synchronous perturbation}

From Theorems 3.1 and 4.1, we have

Theorem 5.1 The degenerate singular point of system $(1.2)_{\delta_{1}=\delta_{2}=0}$ is a fine focus of order nine if and only if the coefficients of its associated complex system $(2.10)_{\delta_{1}=\delta_{2}=0}$ satisfy

$$
\begin{aligned}
& a_{21}=b_{21}=0, \quad a_{30}=-\frac{1}{3} b_{12}, \quad b_{30}=-\frac{1}{3} a_{12}, \\
& -27 a_{03} b_{03}+16 a_{12} b_{12}+216 \lambda=0, \\
& 405 a_{03}^{2} b_{03}^{2}-8,928 a_{03} a_{12} b_{03} b_{12}+6,400 a_{12}^{2} b_{12}^{2}=0, \\
& a_{12}^{2} b_{03}+a_{03} b_{12}^{2}=0, \quad a_{12} b_{12}\left(a_{12}^{2} b_{03}-a_{03} b_{12}^{2}\right) \neq 0 .
\end{aligned}
$$

Theorem 5.2 The infinity of system (1.2) $)_{\delta_{1}=\delta_{2}=0}$ is a fine focus of order seven if and only if the coefficients of its associated complex system $(2.10)_{\delta_{1}=\delta_{2}=0}$ satisfy

$$
\begin{aligned}
& a_{21}=b_{21}=0, \quad a_{30}=-3 b_{12}, \quad b_{30}=-3 a_{12}, \\
& -a_{03} b_{03}+4 a_{12} b_{12}+6 \lambda=0, \quad a_{12} b_{12}\left(a_{12}^{2} b_{03}-a_{03} b_{12}^{2}\right) \neq 0 .
\end{aligned}
$$

Actually, when the degenerate singular point of system $(1.2)_{\delta_{1}=\delta_{2}=0}$ is a fine focus of order nine, its infinity is at most a fine focus of order three; when the infinity of system (1.2) $)_{\delta_{1}=\delta_{2}=0}$ is a fine focus of order seven, its degenerate singular point is at most a fine focus of order three. Therefore, we have

Theorem 5.3 By synchronous perturbation at degenerate singular point and infinity, system (1.2) has the limit cycle configurations of $\{(8), 3\}$ and $\{(3), 6\}$.

For the sake of simplicity, we will prove the first case of Theorem 5.3. The other case can be proved with the same principle.

Theorem 5.4 Let the coefficients in (2.10) satisfy

$$
\begin{aligned}
& \delta_{1}=\delta_{2}=\epsilon_{8}, \quad a_{30}=-\frac{1}{3}, \quad a_{21}=\epsilon_{4}-i \epsilon_{7}, \quad a_{12}=1-\epsilon_{2}-\epsilon_{3}-\epsilon_{5}-i \epsilon_{6}, \\
& a_{03}=-\epsilon_{1}-\frac{4}{3} i \sqrt{\frac{1}{5}(31-2 \sqrt{209})}, \quad \lambda=-\frac{4}{135}(-44+3 \sqrt{209}), \\
& b_{30}=\overline{a_{30}}, \quad b_{21}=\overline{a_{21}}, \quad b_{12}=\overline{a_{12}}, \quad b_{03}=\overline{a_{03}}
\end{aligned}
$$

(accordingly, the coefficients of system (1.2) are determined by (1.4), (2.11) and (5.3)), where $\epsilon_{i}, i=1, \ldots, 8$ are small parameters with

$$
0<\epsilon_{8} \ll \epsilon_{7} \ll \epsilon_{6} \ll \epsilon_{5} \ll \epsilon_{4} \ll \epsilon_{3} \ll \epsilon_{2} \ll \epsilon_{1} \ll 1 .
$$


Then for system (1.2), there are eight limit cycles bifurcating from the degenerate singular point, and at the same time, there are three limit cycles bifurcating from infinity.

Proof For the degenerate singular point of system (1.2), from $\nu_{1}(2 \pi)=e^{2 \pi \delta_{1}}, \nu_{2 m+1}\left(2 \pi, \delta_{1}\right)=$ $\nu_{2 m+1}(2 \pi, 0)+\delta_{1} \phi_{2 m+1}\left(a_{\alpha \beta}, b_{\alpha \beta}, \delta_{1}\right)$, Theorem 3.1 and $\nu_{2 m+1}(2 \pi) \sim i \pi \mu_{m}$, after computing carefully, we have the following:

$$
\begin{aligned}
& \nu_{1}\left(2 \pi, \delta_{1}\right)-1=2 \pi \epsilon_{8}+o\left(\epsilon_{8}\right), \\
& \nu_{3}\left(2 \pi, \delta_{1}\right)=\left[-2 \pi+\omega_{1}\left(\epsilon_{1}, \epsilon_{2}, \epsilon_{3}, \epsilon_{4}, \epsilon_{5}, \epsilon_{6}, \epsilon_{7}\right)\right] \epsilon_{7}+o\left(\epsilon_{7}\right) \text {, } \\
& \nu_{5}\left(2 \pi, \delta_{1}\right)=\left[-\frac{45}{2(-44+3 \sqrt{209})} \pi+\omega_{2}\left(\epsilon_{1}, \epsilon_{2}, \epsilon_{3}, \epsilon_{4}, \epsilon_{5}, \epsilon_{6}, \epsilon_{7}\right)\right] \epsilon_{6}+o\left(\epsilon_{6}\right) \text {, } \\
& \nu_{7}\left(2 \pi, \delta_{1}\right)=\left[-\frac{1,215 \sqrt{5(31-2 \sqrt{209})}}{8(-44+3 \sqrt{209})^{2}} \pi+\omega_{3}\left(\epsilon_{1}, \epsilon_{2}, \epsilon_{3}, \epsilon_{4}, \epsilon_{5}, \epsilon_{6}, \epsilon_{7}\right)\right] \epsilon_{5}+o\left(\epsilon_{5}\right) \text {, } \\
& \nu_{9}\left(2 \pi, \delta_{1}\right)=\left[-\frac{54,675 \sqrt{5(31-2 \sqrt{209})}}{8(-44+3 \sqrt{209})^{3}} \pi+\omega_{4}\left(\epsilon_{1}, \epsilon_{2}, \epsilon_{3}, \epsilon_{4}, \epsilon_{5}, \epsilon_{6}, \epsilon_{7}\right)\right] \epsilon_{4}+o\left(\epsilon_{4}\right) \text {, } \\
& \nu_{11}\left(2 \pi, \delta_{1}\right)=\left[-\frac{273,375 \sqrt{5(31-2 \sqrt{209})}}{4(-44+3 \sqrt{209})^{4}} \pi+\omega_{5}\left(\epsilon_{1}, \epsilon_{2}, \epsilon_{3}, \epsilon_{4}, \epsilon_{5}, \epsilon_{6}, \epsilon_{7}\right)\right] \epsilon_{3}+o\left(\epsilon_{3}\right) \text {, } \\
& \nu_{15}\left(2 \pi, \delta_{1}\right)=\left[\frac{22,143,375 \sqrt{5(31-2 \sqrt{209})}(-418+31 \sqrt{209})}{16(-44+3 \sqrt{209})^{6}} \pi\right. \\
& \left.+\omega_{6}\left(\epsilon_{1}, \epsilon_{2}, \epsilon_{3}, \epsilon_{4}, \epsilon_{5}, \epsilon_{6}, \epsilon_{7}\right)\right] \epsilon_{2}+o\left(\epsilon_{2}\right) \\
& \nu_{17}\left(2 \pi, \delta_{1}\right)=\left[-\frac{174,379,078,125 \sqrt{5(31-2 \sqrt{209})}(-29+2 \sqrt{209})}{1,024(-44+3 \sqrt{209})^{7}} \pi\right. \\
& \left.+\omega_{7}\left(\epsilon_{1}, \epsilon_{2}, \epsilon_{3}, \epsilon_{4}, \epsilon_{5}, \epsilon_{6}, \epsilon_{7}\right)\right] \epsilon_{1}+o\left(\epsilon_{1}\right) \\
& \nu_{19}\left(2 \pi, \delta_{1}\right)=-\frac{32,882,911,875 \sqrt{5(31-2 \sqrt{209})}(-720,929+49,818 \sqrt{209})}{7,168(-44+3 \sqrt{209})^{8}} \pi+o(1) \text {, }
\end{aligned}
$$

where $\omega_{i}\left(\epsilon_{1}, \epsilon_{2}, \epsilon_{3}, \epsilon_{4}, \epsilon_{5}, \epsilon_{6}, \epsilon_{7}\right)$ are analytic at $(0,0,0,0,0,0,0)$ and $\omega_{i}(0,0,0,0,0,0,0)=0$, $i=1, \ldots, 7$.

Simultaneously, for the infinity of system (1.2), we have

$$
\begin{aligned}
& \nu_{1}\left(2 \pi, \delta_{2}\right)-1=-2 \pi \epsilon_{8}+o\left(\epsilon_{8}\right), \\
& \nu_{3}\left(2 \pi, \delta_{2}\right)=2 \pi \epsilon_{7}+o\left(\epsilon_{7}\right), \\
& \nu_{5}\left(2 \pi, \delta_{2}\right)=-\frac{2}{3} \pi \epsilon_{6}+o\left(\epsilon_{6}\right), \\
& \nu_{7}\left(2 \pi, \delta_{2}\right)=\frac{16}{9} \sqrt{\frac{1}{5}(31-2 \sqrt{209})} \pi+o(1) .
\end{aligned}
$$

According to the classical theory of Bautin, there exist eight limit cycles in a small enough neighborhood of the degenerate singular point and three limit cycles in a small enough neighborhood of the infinity. 


\section{Concluding remarks}

We have presented the solution to the problem of bifurcation of limit cycles at degenerate singular point and infinity for a class of seventh degree polynomial vector field. There are two important points to make here. Firstly, by computing the first several singular point quantities with Mathematica and vanishing them, the same center conditions at degenerate singular point (Theorem 3.2) and at infinity (Theorem 4.2) are obtained. Secondly, by synchronous perturbation of degenerate singular point and infinity, the limit cycle configurations of $\{(8), 3\}$ and $\{(3), 6\}$ are determined. We hope that our work could make some contributions in this direction.

\section{Competing interests}

The authors declare that they have no competing interests.

\section{Authors' contributions}

The study was carried out in collaboration between both of the authors. YW completed the main part of this paper, FL corrected the main theorems and polished the manuscript. All authors read and approved the final manuscript.

\section{Author details}

'School of Mathematics and Statistics, Henan University of Science and Technology, Luoyang, Henan 471003, P.R. China.

${ }^{2}$ School of Science, Linyi University, Linyi, Shandong 276005, P.R. China.

\section{Acknowledgements}

Acknowledgments are due to the referees for their useful suggestions and valuable comments. This work is supported in part by the National Nature Science Foundation of China (No. 11101126, 11126114 and 11201211), the Fund of Henan Education Commission (No. 2011B110014), and the Scientific Research Foundation for Doctoral Scholars of Haust (09001524).

Received: 5 October 2012 Accepted: 18 December 2012 Published: 9 January 2013

\section{References}

1. Li, J: Hilbert's 16th problem and bifurcations of planar polynomial vector fields. Int. J. Bifurc. Chaos 13, 47-106 (2003)

2. Ye, Y: Qualitative Theory of Polynomial Differential Systems. Shanghai Sci. Technol., Shanghai (1995) (in Chinese)

3. Liu, Y: Theory of center-focus for a class of higher-degree critical points and infinite points. Sci. China Ser. A 44, 37-48 (2001)

4. Blows, TR, Rousseau, C: Bifurcation at infinity in polynomial vector fields. J. Differ. Equ. 104, 215-242 (1993)

5. Liu, Y, Chen, H: Stability and bifurcations of limit cycles of the equator in a class of cubic polynomial systems. Comput. Math. Appl. 44, 997-1005 (2002)

6. Liu, Y, Huang, W: Seven large-amplitude limit cycles in a cubic polynomial system. Int. J. Bifurc. Chaos 16, 473-485 (2006)

7. Zhang, Q, Liu, Y: A cubic polynomial system with seven limit cycles at infinity. Appl. Math. Comput. 177, $319-329$ (2006)

8. Liu, Y, Zhao, M: Stability and bifurcation of limit cycles of the equator in a class of fifth polynomial systems. Chin. J. Contemp. Math. 23, 75-78 (2002)

9. Chen, H, Liu, Y: Limit cycle of the equator in a quintic polynomial system. Chin. J. Contemp. Math. Ser. A 24, 219-224 (2003) (in Chinese)

10. Huang, W, Liu, Y: Bifurcations of limit cycles from infinity for a class of quintic polynomial system. Bull. Sci. Math. 128 291-302 (2004)

11. Zhang, Q, Liu, Y: A quintic polynomial differential system with eleven limit cycles at the infinity. Comput. Math. Appl. 53, 1518-1526 (2007)

12. Huang, W, Liu, Y: A polynomial differential system with eight limit cycles at infinity. J. Math. (Wuhan) 24(5), 551-556 (2004)

13. Huang, W, Liu, Y: A polynomial differential system with nine limit cycles at infinity. Comput. Math. Appl. 48, 577-588 (2004)

14. Zhang, Q, Gui, W, Liu, Y: Bifurcation of limit cycles at the equator for a class of polynomial differential system. Nonlinear Anal., Real World Appl. 10, 1042-1047 (2009)

15. Huang, W, Liu, Y, Tang, Q: Singular point quantities and bifurcations of limit cycles for a class of quintic polynomial systems. Acta Math. Sci. Ser. A 25, 744-752 (2005) (in Chinese)

16. Zhang, Q, Liu, Y, Chen, H: Bifurcation at the equator for a class of quintic polynomial differential system. Appl. Math. Comput. 181, 747-755 (2006)

17. Liu, Y, Li, J: Theory of values of singular point in complex autonomous differential system. Sci. China Ser. A 3, 10-24 (1990)

18. Chen, H, Liu, Y, Zeng, X: Algebraic recursion formulas for quantities of equator in a planar polynomial differential system. Acta Math. Sin. 48, 963-972 (2005) (in Chinese) 